\title{
Acute rhinosinusitis - are we forgetting the possibility of a dental origin? A retrospective study of 385 patients
}

\section{Wuokko-Landen, Annina}

2019-09-02

Wuokko-Landen , A , Blomgren , K \& Välimaa , H 2019 , ' Acute rhinosinusitis - are we forgetting the possibility of a dental origin? A retrospective study of 385 patients ' , Acta Oto-Laryngologica , vol. 139 , no. 9 , pp. 783-787 . https://doi.org/10.1080/00016489.2019.1634837

http://hdl.handle.net/10138/317311

https://doi.org/10.1080/00016489.2019.1634837

acceptedVersion

Downloaded from Helda, University of Helsinki institutional repository.

This is an electronic reprint of the original article.

This reprint may differ from the original in pagination and typographic detail.

Please cite the original version. 


\title{
Acute rhinosinusitis - Are we forgetting the possibility of a dental origin? A retrospective study of 385 patients
}

\author{
Annina Wuokko-Landén ${ }^{1}$, Karin Blomgren², Hannamari Välimaa ${ }^{3,4}$ \\ ${ }^{1}$ Faculty of Medicine, University of Helsinki, Helsinki, Finland, ${ }^{2}$ Department of \\ Otorhinolaryngology, University of Helsinki and Helsinki University Hospital, Helsinki,
} Finland, ${ }^{3}$ Department of Virology, University of Helsinki, Helsinki, Finland,

${ }^{4}$ Department of Oral and Maxillofacial Surgery, University of Helsinki and Helsinki

University Hospital, Helsinki, Finland

\section{CORRESPONDING AUTHOR}

Annina Wuokko-Landén

Doctoral School of Health Sciences

Faculty of Medicine

University of Helsinki, Helsinki, Finland

annina.wuokko@ fimnet.fi, tel.+358505305031

Karin Blomgren

Department of Otorhinolaryngology

Helsinki University Hospital

P.O. BOX 263

FI-00029 HUS, Helsinki, Finland

Hannamari Välimaa

Department of Virology

University of Helsinki

P.O. BOX 21

00014 University of Helsinki, Helsinki, Finland 


\section{Acute rhinosinusitis - Are we forgetting the possibility of a dental origin? A retrospective study of 385 patients}

Background: Odontogenic sinusitis (OS) is a common but underdiagnosed form of acute rhinosinusitis (ARS). OS carries no specific characteristics, but unilateral symptoms and certain microbiological as well as radiological findings indicate odontogenic origin.

Aims/Objectives: We studied the proportion of OS in ARS patients, the presence and associations of unilateral symptoms, and possible OS microbial and radiological findings. In addition, we investigated how this condition is recognised among ear, nose, and throat specialists and radiologists.

Materials and methods: All 676 ARS patients treated in the Department of Otorhinolaryngology at Helsinki University Hospital in 2013 were retrospectively enrolled. The data was collected from patients' hospital medical records, the laboratory database, and radiological reports.

Results: Odontogenic origin of ARS was suspected in 59 (15.3\%) patients. Altogether (29.9\%) 115 patients complained of unilateral symptoms and these were found to associate with probable oral microbial findings ( $\mathrm{p}<0.001)$. These findings covered $20.2 \%$ of isolates. Teeth were mentioned in $89.6 \%$ of the radiological reports.

Conclusions and Significance: Odontogenic sinusitis is common among patients with ARS, and good diagnostic tools already exist in routine practice. Microbial and radiological findings should be carefully evaluated, especially in cases of unilateral symptoms.

Key Words: sinusitis; maxillary sinus; paranasal sinuses; diagnosis; periapical diseases; dental pulp diseases; iatrogenic disease 


\section{Introduction}

Acute rhinosinusitis (ARS) is either a continuum of a viral upper respiratory infection or bacterial infection [1]. Because the maxillary teeth are located close to the maxillary sinus, ARS may also arise from an odontogenic source (odontogenic sinusitis, OS). Odontogenic infections are a known cause of sinusitis, but often overlooked [2] OS may follow periapical or periodontal dental infection, pericoronitis, or dental implant infection. Iatrogenic factors, such as foreign bodies in the sinus and a non-closing oroantral communication following a tooth extraction, can also result in OS [3]. Odontogenic infection can spread further from the maxillary sinus into the other paranasal sinuses and then opacification of the ethmoids is often present [4].

Estimates of OS incidence vary depending on study protocols and diagnostic criteria. Perhaps the most commonly cited incidence, $10 \%$ to $12 \%$ of all rhinosinusitis, lacks good evidence, however, and the source data dates back over 50 years [5]. Larger estimates also exist, rising up to $40 \%$ of all maxillary sinusitis cases [6]. In a Finnish national health examination survey of over 5000 dentate adults, dental periapical lesions correlated with sinus mucosa thickening [7].

The traditionally mentioned signs of OS are unilateral symptoms, foul-smelling discharge and pain in the maxillary teeth, but none of these is specific for OS [2]. The most common pathogens in ARS are Streptococcus pneumoniae, Haemophilus influenzae, Moraxella catarrhalis, and Staphylococcus aureus [8]. As for the OS, a mixed polymicrobial infection with anaerobes outnumbering aerobes is considered a typical finding [9].

Diagnosis of OS requires radiological imaging, however, plain dental radiographs often fail in evaluating periapical disease in maxillary teeth and thus may easily lead to false negative findings [10]. Computed tomography (CT) and cone-beam 
computed tomography (CBCT) often serve to evaluate sinus conditions. CBCT can provide considerable amount of additional clinically relevant dental information not apparent in the plain dental radiographs [10], but when diagnosing rhinosinusitis, the teeth are often neglected [2].

In the present study, our aim was to investigate an estimated proportion of OS in ARS patients and how well this condition is recognized among ear, nose, and throat (ENT) residents, specialists and radiologists at the secondary and tertiary healthcare level. We also studied the presence and associations of unilateral symptoms and possible OS microbial and radiological findings.

\section{Materials and methods}

All patients (676) who visited the Department of Otorhinolaryngology at the Helsinki University Hospital (HUH) in 2013 because of ARS were retrospectively enrolled. Clinical examination was performed by an ENT resident or by an ENT specialist. Patients with the ICD-10 J01 subcodes for acute maxillary (J01.0), frontal (J01.1), ethmoidal (J01.2), other (J01.8), or uncategorized sinusitis (J01.9) and pansinusitis (J01.4) were included, with the exception of isolated frontal sinusitis. Each patient had one or more of these codes.

Patients who first visited the HUH clinic in 2013, and that were reported as having no history of previous sinus surgery, were evaluated. A total of 385 ARS patients met these inclusion criteria. Only patient record entries from the year 2013 were included.

The data was collected from patient hospital medical records, the laboratory database, and radiological reports. The data included age, sex, smoking status, allergies, diseases, and pregnancy. Microbial findings were recorded from routine diagnostic bacterial 
samples cultured in both aerobic and anaerobic conditions at Helsinki University Hospital Laboratory Services (HUSLAB). The samples for cultures were taken either by antral irrigation or in conjunction with maxillary sinus surgery. Only the first sample was taken into account.

A count was made of suspected OS cases, the number of patients advised to visit a dentist, the number of times dentition was mentioned, and the frequency of dental examinations. Only the radiological reports of sinus CT, CBCT, and panoramic tomography (PTG) examinations were studied. The number of pathological maxillary dental findings, according to the radiological reports, were recorded.

\section{Statistical methods}

The associations between the variables were analysed with chi-square test or Fisher's exact test. The modifying effect of the factors (smoking, allergies, asthma, diseases predisposing to infection, lower respiratory system disease, simultaneous pneumonia, or pregnancy) on the association between typical oral microbial findings and unilateral symptoms was tested using binary logistic regression for interaction effect between potential modifying factor and microbial finding. Statistical analyses were performed with IBM SPSS Statistics, version 24.0 (IBM Corp., Armonk, NY). P-values less than 0.05 were considered statistically significant.

\section{Ethical considerations}

All procedures performed in the study are in accordance with the ethical standards of the Finnish legislation. Our study has the approval of the Head of the Department of Otorhinolaryngology at HUH and due to the nature of the study, no informed consents were obtained from the study patients. 


\section{Results}

Of the 676 patients who visited the HUH Department of Otorhinolaryngology during 2013 because of ARS, a total of 385 met the inclusion criteria. Background data of the patients is presented in Table 1.

Unilateral symptom complaints were made by 115 patients $(29.9 \%)$. OS was strongly suspected in 14 patients (3.6\%) and suspected in 45 patients (11.7\%). Of the strongly suspected OS patients and suspected OS patients, 12/14 and 21/45 had unilateral symptoms, respectively. The status of the teeth was overlooked entirely in 219 patients (56.9\%) (Table 2).

\section{Microbial findings}

The vast majority of the patients $(350 / 385,90.9 \%)$ underwent antral irrigation. A total of 291 patients $(75.6 \%)$ had a microbial sample taken, of which $240(82.5 \%)$ showed microbial growth. One sample with growth had on average 1.4 microbial isolates. Seventy of the 342 isolates (20.5\%) represented probable oral microbial findings: 24 (7.0\%) were aerobes typical for oral microbiota and 44 (12.9\%) anaerobes typical for oral microbiota (Table 3). In addition, one Candida albicans and one Candida dubliniensis isolate were considered typical oral microbial findings. Of all patients, including those without microbial samples, $61.6 \%$ had aerobic and $11.2 \%$ anaerobic microbial findings.

A mixed aerobe-anaerobe growth was present in $16 \%$ of the samples, and $88.4 \%$ of the samples containing anaerobes also had aerobe microbes. The presence of anaerobic gram-negative rods was associated with the Streptococcus anginosus group (p

$<0.001$ ) (Table 4). There were no associations between other bacterial subgroups or 
species. Bacteria typical for oral flora associated with unilateral symptoms (aerobes $\mathrm{p}=$ 0.001 , anaerobes $\mathrm{p}<0.001)$ (Table 5).

\section{Radiological examinations}

CTs or CBCTs were taken from 59 and PTGs from 17 patients. Three patients had either CT or CBCT simultaneously with PTG radiograph. Figure 1 demonstrates CT scan views of one strongly suspected OS patient and one ARS patient without suspected odontogenic cause. Some radiographs taken in the referring unit lacked a report, leaving 67 radiological reports of the 76 radiographs available. Of the reports, $60(89.6 \%)$ mentioned maxillary teeth and almost half of the reports $(29 / 67,43.3 \%)$ indicated pathological findings in maxillary teeth or supporting tissues. All PTG reports and 45 (86.5\%) of the CT and CBCT reports mentioned maxillary teeth. In 23 of the 29 cases (79.3\%) the reported pathological dental or supporting tissue findings were taken into account while planning or providing treatment for ARS.

Unilateral symptoms were not associated with pathological radiological findings in maxillary teeth. Pathological radiological findings were associated with anaerobic bacterial findings typical for oral microbiota $(p=0.039)($ Table 6$)$.

\section{Discussion}

The ARS of a notable percentage (15.3\%) of the 385 patients in this study was of possible odontogenic origin. Previous estimates of OS incidence vary mostly between $10 \%$ and $40 \%$, yet only a minority of guidelines worldwide even mention odontogenic infections as a cause of rhinosinusitis [5]. Lack of well-guided treatment paths and of adequate knowledge can result in underdiagnosis and undertreatment of this common disease. Here also, teeth were not routinely mentioned (43.1\%) and they were rarely examined $(8.1 \%)$. Lack of dental pain or recent dental operations should not delay an 
OS diagnosis suspicion, because dental pain is often absent and the origins of OS are diverse [11]. Disease-causing iatrogenic dental operations may have taken place far in the past, and a previous appointment with a dentist does neither exclude the possibility of OS nor even dental infection.

No symptom is shown to be unique to OS, but in a recent study, as many as $72.6 \%$ of unilateral sinusitis cases were of odontogenic origin [12]. Almost one third (29.9\%) of our study population complained of unilateral symptoms, which showed an association $(\mathrm{p}<0.001)$ with probable oral aerobe and anaerobe microbial findings in the sinus samples. When coexisting, these findings may serve as a tool for OS diagnosis.

Most of our patients underwent maxillary irrigation, which provided us with information about the sinus microbial condition. Understanding the difference between the microbiology of ARS and OS is important, and microbiological diagnosis is essential for choosing the correct treatment and medication as well. Samples taken endoscopically from the middle meatus correspond to the maxillary sinus aspirates [13] so the swab from the middle meatus is also useful. The appropriate antimicrobial medication may limit the spread of odontogenic infection into the sinus, but a complete cure demands dental treatment. Some micro-organisms are present in the undisturbed, healthy sinus, where the active epithelium and mucociliary clearance prevent significant colonization [14]. In our study, $20.5 \%$ of all the isolates from the maxillary sinus samples were typical microbes of the oral cavity, suggesting that some oral bacteria can penetrate the sinus mucosa and cause inflammation leading to ARS. Despite that, rhinosinusitis symptoms and dental pain can be minimal or absent as long as no osteomeatal obstruction occurs [3].

After a sterile dental pulp is infected, the root canal system is colonized with a mixture of bacteria, mostly anaerobes, and when these bacteria spread into the 
periapical region and further into the jaw, dentoalveolar abscesses may develop [15]. Our most common findings among anaerobes and aerobes of suspected oral origin were gram-negative rods and Viridans group streptococci, respectively. The very same bacteria, here found in maxillary sinus samples, are also the most common findings in dentoalveolar abscesses [15]. Previously, anaerobic gram-negative rods and aerobic gram-positive cocci have also been linked to both acute and chronic OS [9]. We also found an association $(\mathrm{p}<0.001)$ of the anaerobic gram-negative rods with the Streptococcus anginosus group. Therefore, when these species coexist, a dental infection should be strongly suspected.

Anaerobe-containing samples also had aerobes in $88.4 \%$ of cases, and $16 \%$ of all samples had a mixed aerobe-anaerobe growth, which is typical in oral infections. It seems that Streptococcus pneumoniae, Haemophilus influenzae, and Moraxella catarrhalis do not generally appear in OS [9] and we found no association with these to other bacteria. Staphylococcus aureus has been a major finding and a suspected pathogen in both OS [16] and ARS [8], but we found it in only 5.5\% of the samples.

Radiological signs of sinusitis are associated with dental pathology [7] and when radiographic findings of ARS are severe, odontogenic infections are very often the source of the disease [17] OS might be present with a healthy dental examination result, mostly because of the limitations of the dental radiographs that are routinely used [18]. Neglect of the teeth and surrounding tissues seems to be common in radiological examination reports $[19,20]$. Seven of our 76 reports also failed to make observations of the teeth, and in five cases the reported pathological dental findings were not taken into account in the treatment. In our study, pathological findings mentioned in the radiological reports were not associated with unilateral symptoms, but that might be due to the small percentage of patients with PTG or CT, or to the CBCT scans available. 
Pathological radiological findings were, on the other hand, associated with typical oral anaerobe findings.

Our study, like many studies concerning OS, has some limitations. Our aim was to investigate the already existing manner of OS diagnostics, which demanded retrospective study design. The observations are based on patient medical records which may be insufficient and vary depending on the ENT specialist or resident in question, thus causing bias. Because the suspected OS patients were referred to or recommended to visit another clinic for dental treatment and often radiography, we could not verify these OS diagnoses. Thus, the number of possible OS patients reflects more the level of diagnostics than the exact amount of OS patients. Furthermore, only a minority of these ARS patients had radiological examinations, where a possible teeth pathology could be observed. Microbial sampling techniques, antimicrobial medication, and sinus normal flora confound microbial results. Additionally, patients were referred from primary health care, so the mildest cases of ARS are probably not represented in our material. Despite these issues our study population represents a large, authentic group of ARS patients and their diagnostics at the tertiary health care level.

Broad, prospective studies in collaboration with ENTs, radiologists, and dentists are needed in order to create good diagnostic and treatment paths and to prevent the underdiagnosing of OS. As was shown in this study, many diagnostic tools already exist in our routine practice, and their results should be carefully evaluated when treating ARS. Ideally, many OS patients could be recognized already by doctors at the primary health care level to avoid unnecessary referrals. The possibility of odontogenic infection should always be considered when diagnosing rhinosinusitis.

\section{Conclusions}

Odontogenic infection is a common origin of ARS, as suggested in our study, where 
$15.3 \%$ of ARS diagnoses were connected to possible dental causes. Unilateral symptoms and typical oral bacterial findings in maxillary sinus cultures show an association, and their concurrent presence can be a sign of OS. Attention to the teeth and referrals to dental professionals are not routinely performed when suspecting OS, and dental findings in radiographs are sometimes neglected. Thus, collaboration between doctors, ENTs, radiologists, and dentists is essential for timely and accurate diagnosis and treatment of OS.

\section{Acknowledgements}

This work was supported by the Helsinki University Hospital Research Fund. The statistical analysis was performed by statistician Tero Vahlberg, and we thank adjunct professor Tuomas Klockars for his help in revising the manuscript.

\section{Disclosure of interest}

The authors report no conflict of interest.

\section{References}

1. Autio TJ, Tapiainen T, Koskenkorva T, et al. The role of microbes in the pathogenesis of acute rhinosinusitis in young adults. Laryngoscope 2015;125(1):E1-7.

2. Patel NA, Ferguson BJ. Odontogenic sinusitis: an ancient but under-appreciated cause of maxillary sinusitis. Curr Opin Otolaryngol Head Neck Surg 2012;20(1):24-28.

3. Brook I. Sinusitis of odontogenic origin. Otolaryngol Head Neck Surg 2006;135(3):349-355.

4. Saibene AM, Pipolo GC, Lozza P, et al. Redefining boundaries in odontogenic sinusitis: a retrospective evaluation of extramaxillary involvement in 315 patients. Int Forum Allergy Rhinol 2014;4(12):1020-1023.

5. Longhini AB, Ferguson BJ. Clinical aspects of odontogenic maxillary sinusitis: a case series. Int Forum Allergy Rhinol 2011;1(5):409-415.

6. Melen I, Lindahl L, Andreasson L, et al. Chronic maxillary sinusitis. Definition, diagnosis and relation to dental infections and nasal polyposis. Acta Otolaryngol 1986;101(3-4):320-327.

7. Vallo J, Suominen-Taipale L, Huumonen S, et al. Prevalence of mucosal abnormalities of the maxillary sinus and their relationship to dental disease in panoramic radiography: results from the Health 2000 Health Examination Survey. Oral Surg Oral Med Oral Pathol Oral Radiol Endod 2010;109(3):e80-7. 
8. Payne SC, Benninger MS. Staphylococcus aureus is a major pathogen in acute bacterial rhinosinusitis: a meta-analysis. Clin Infect Dis 2007;45(10):e121-7.

9. Brook I. Microbiology of acute and chronic maxillary sinusitis associated with an odontogenic origin. Laryngoscope 2005;115(5):823-825.

10. Shahbazian M, Vandewoude $\mathrm{C}$, Wyatt $\mathrm{J}$ et al. Comparative assessment of panoramic radiography and $\mathrm{CBCT}$ imaging for radiodiagnostics in the posterior maxilla. Clin Oral Investig 2014;18(1):293-300.

11. Zirk M, Dreiseidler T, Pohl M, et al. Odontogenic sinusitis maxillaris: A retrospective study of 121 cases with surgical intervention. J Craniomaxillofac Surg 2017;45(4):520-525.

12. Matsumoto Y, Ikeda T, Yokoi H, et al. Association between odontogenic infections and unilateral sinus opacification. Auris Nasus Larynx 2015;42(4):288-293.

13. Vogan JC, Bolger WE, Keyes AS. Endoscopically guided sinonasal cultures: a direct comparison with maxillary sinus aspirate cultures. Otolaryngol Head Neck Surg 2000;122(3):370-373.

14. Tucker MR, Schow SR. Odontogenic Diseases of the Maxillary Sinus. In: Hupp JR, Ellis EI, Tucker MR, editors. Contemporary Oral and Maxillofacial Surgery. St. Louis, Missouri: Mosby Elsevier; 1988. p. 383-395.

15. Robertson D, Smith AJ. The microbiology of the acute dental abscess. J Med Microbiol 2009 Feb;58(Pt 2):155-162.

16. Saibene AM, Vassena C, Pipolo C, et al. Odontogenic and rhinogenic chronic sinusitis: a modern microbiological comparison. Int Forum Allergy Rhinol 2016;6(1):41-45.

17. Bomeli SR, Branstetter BF 4th, Ferguson BJ. Frequency of a dental source for acute maxillary sinusitis. Laryngoscope 2009;119(3):580-584.

18. Longhini AB, Branstetter BF, Ferguson BJ. Unrecognized odontogenic maxillary sinusitis: a cause of endoscopic sinus surgery failure. Am J Rhinol Allergy 2010;24(4):296-300.

19. Wang KL, Nichols BG, Poetker DM, et al. Odontogenic sinusitis: a case series studying diagnosis and management. Int Forum Allergy Rhinol 2015;5(7):597-601.

20. Longhini AB, Branstetter BF, Ferguson BJ. Otolaryngologists' perceptions of odontogenic maxillary sinusitis. Laryngoscope 2012;122(9):1910-1914 
Figure 1. Coronal views of two computed tomography scans. (A) Strongly suspected odontogenic sinusitis (OS) with a large apical periodontis (white arrow) around the first maxillary molar roots on the left and opacified left maxillary sinus. Besides having Streptococcus anginosus group and anaerobic gram-negative rod microbial findings, the patient complained unilateral symptoms. (B) Acute rhinosinusitis without OS suspicion. Comprehensive maxillary sinus opacification with no pathology in the maxillary teeth. Symptoms were bilateral and microbial finding was coagulase-negative staphylococci. 


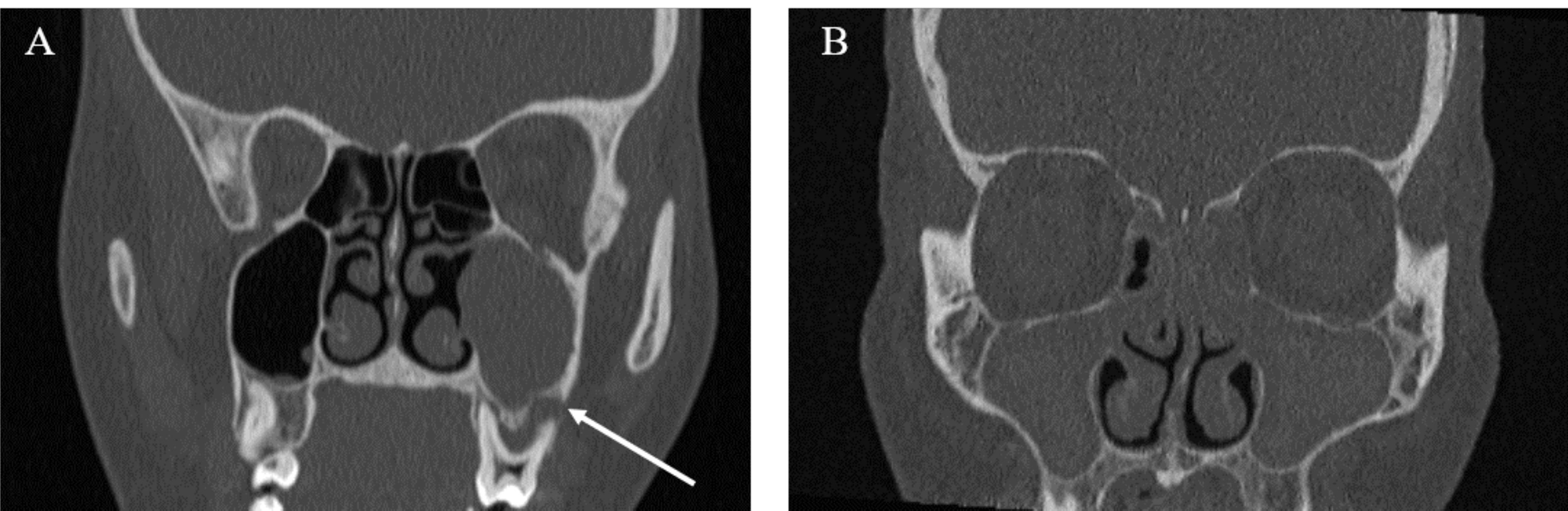


Table 1. Patient characteristics $(\mathrm{N}=385)$.

n $(\%)$

\begin{tabular}{lc}
\hline Gender & \\
Female & $243(63.1)$ \\
Male & $142(36.9)$ \\
\hline Mean age in years (SD) & $41.7(17.7)$ \\
\hline Smoking & $84(21.8)$ \\
\hline Allergies & $122(31.7)$ \\
\hline Asthma & $63(16.4)$ \\
\hline Disease predisposing to infections & $65(16.9)$ \\
\hline Disease of lower respiratory tract other than asthma & $11(2.9)$ \\
\hline Simultaneous pneumonia & $28(7.3)$ \\
\hline Pregnancy & $21(5.5)$
\end{tabular}


Table 2. Possible OS diagnoses and oral and dental observations in ARS patients $(\mathrm{N}=385)$ according to ENT specialists or residents.

\begin{tabular}{lc} 
& $\mathrm{n}(\%)$ \\
\hline Possible OS diagnosis & $59(15.3)$ \\
Suspected OS diagnosis & $45(11.7)$ \\
$\quad$ Suspected OS and patient referred to or recommended to visit a dentist & $36(9.4)$ \\
$\quad$ Suspected OS without further actions & $9(2.3)$ \\
Strongly suspected OS diagnosis & $14(3.6)$ \\
\hline Teeth mentioned & $166(43.1)$ \\
Teeth examined & $31(8.1)$ \\
Teeth not mentioned & $219(56.9)$ \\
\hline Oral mucosa examined & $329(85.5)$
\end{tabular}

ARS = acute rhinosinusitis

OS = odontogenic sinusitis 
Table 3. Microbial isolates in 291 maxillary sinus samples.

\begin{tabular}{|c|c|c|}
\hline & $\mathrm{n}(\%$ of 342 isolates) & n (\% of 291 samples) \\
\hline Aerobes & $294(86.0)$ & $237(81.4)$ \\
\hline Gram-positive cocci & $131(38.3)$ & $124(42.6)$ \\
\hline Alpha-hemolytic streptococci & $74(21.6)$ & $74(25.4)$ \\
\hline Streptococcus pneumoniae & $52(15.2)$ & $52(17.9)$ \\
\hline Viridans group streptococci $\dagger$ & $22(6.4)$ & $22(7.6)$ \\
\hline Streptococcus anginosus group $\dagger$ & $21(6.1)$ & $21(7.2)$ \\
\hline Not specified $\dagger$ & $1(0.29)$ & $1(0.3)$ \\
\hline Staphylococci & $49(14.3)$ & $47(16.2)$ \\
\hline Coagulase-negative staphylococci & $33(9.6)$ & $33(11.0)$ \\
\hline Staphylococcus aureus & $16(4.7)$ & $16(5.5)$ \\
\hline Beta-hemolytic streptococci & $7(2.0)$ & $7(2.4)$ \\
\hline Streptococcus pyogenes & $4(1.2)$ & $4(1.4)$ \\
\hline Streptococcus betahemolyticus & $3(0.9)$ & $3(1.0)$ \\
\hline Enterococci & $1(0.29)$ & $1(0.34)$ \\
\hline Enterococcus faecium $\dagger$ & $1(0.29)$ & $1(0.34)$ \\
\hline Other aerobes (mostly normal flora) & $88(25.7)$ & $86(29.6)$ \\
\hline Eikenella corrodens $\dagger$ & $1(0.29)$ & $1(0.3)$ \\
\hline Gram-negative rods & $75(21.9)$ & $72(24.7)$ \\
\hline Other gram-negative rods & $50(14.6)$ & $49(16.8)$ \\
\hline Haemophilus influenzae & $44(12.9)$ & $44(15.1)$ \\
\hline Moraxella catarrhalis & $6(1.8)$ & $6(2.1)$ \\
\hline Coliform rods & $23(6.7)$ & $23(7.9)$ \\
\hline Pseudomonas-like gram-neg. rod & $2(0.6)$ & $2(0.7)$ \\
\hline Anaerobes & $46(13.5)$ & $43(14.8)$ \\
\hline Gram-negative rods $\dagger$ & $33(9.6)$ & $33(11.3)$ \\
\hline Prevotella species $\dagger$ & $7(2.0)$ & $7(2.4)$ \\
\hline Fusobacterium necrophorum $\dagger$ & $1(0.29)$ & $1(0.3)$ \\
\hline Not specified $\dagger$ & $25(7.3)$ & $25(8.6)$ \\
\hline
\end{tabular}




\begin{tabular}{lll} 
Anaerobic mixed growth $\dagger$ & $6(1.8)$ & $6(2.1)$ \\
Gram-positive cocci $\dagger$ & $5(1.5)$ & $5(1.7)$ \\
Parvimonas micra $\dagger$ & $2(0.6)$ & $2(0.7)$ \\
Peptostreptococcus anaerobius $\dagger$ & $1(0.29)$ & $1(0.3)$ \\
Not specified $\dagger$ & $2(0.6)$ & $2(0.6)$ \\
Gram-positive rods & $2(0.6)$ & $2(0.7)$ \\
Not specified & $2(0.6)$ & $2(0.7)$ \\
\hline Fungi & $2(0.6)$ & $2(0.7)$ \\
\hline Candida species $\dagger$ & $2(0.6)$ & $2(0.7)$
\end{tabular}

$\dagger$ Microbial findings typical for oral microbiota 
Table 4. Association of the Streptococcus anginosus group with anaerobic gram-negative rods.

Streptococcus anginosus group

\begin{tabular}{llll}
\hline & No & Yes & p-value \\
\hline Anaerobic gram-negative rods & & $\mathrm{n}(\%)$ & \\
\hline No & & & $<0.001$ \\
\hline Yes & $251(93.0)$ & $7(33.3)$ & \\
\hline
\end{tabular}


Table 5. Association of unilateral symptoms with aerobic and anaerobic bacteria typical for oral microbiota. Unilateral symptoms

\begin{tabular}{llll}
\hline & No & Yes & p-value \\
\hline & $\mathrm{n}(\%)$ & $\mathrm{n}(\%)$ & \\
\hline Aerobes typical for oral microbiota & & & $<0.001$ \\
& & & \\
\hline No & $191(71.5)$ & $76(28.5)$ & \\
\hline Yes & $9(37.5)$ & $15(62.5)$ & \\
\hline Anaerobes typical for oral microbiota & & & \\
\hline No & $182(72.8)$ & $68(27.2)$ & \\
\hline Yes & $18(43.9)$ & $23(56.1)$ & \\
\hline
\end{tabular}


Table 6. Association of pathological dental findings in maxillary teeth with typical oral aerobic and anaerobic bacteria.

Pathological radiological findings in maxillary teeth

\begin{tabular}{llll}
\hline No & Yes & p-value \\
\hline $\mathrm{n}(\%)$ & $\mathrm{n}(\%)$
\end{tabular}

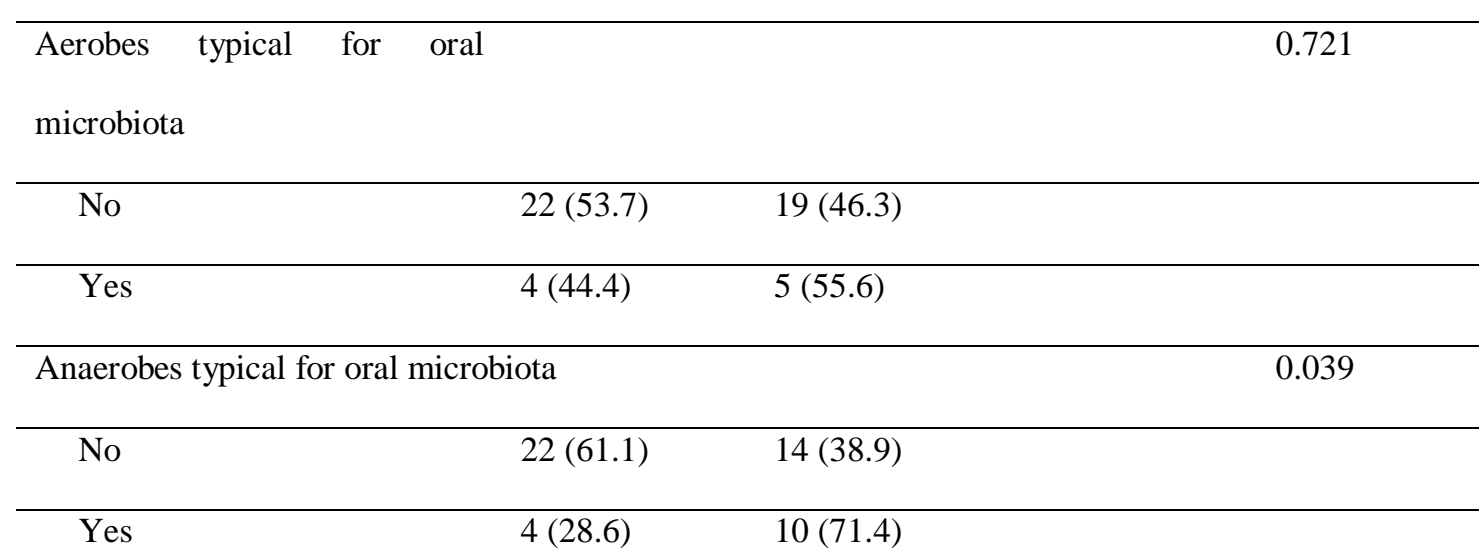

